GPPS-TC-2019-0088

\title{
NUMERICAL SIMULATION OF GAS TURBINE BURNING CHAMBER COMBUSTION WITH DEFINED BURNER DEFECTS
}

\author{
Christoph Hennecke
}

\author{
Henrik von der Haar* \\ vonderhaar@itv.uni-hannover.de \\ Institute of Technical Combustion \\ Leibniz University Hannover \\ Hannover, Germany
}

\author{
Friedrich Dinkelacker
}

lengthened or shortened depending on the actual engine state instead of prescribed maintenance intervals. Additionally, the actual regeneration process could be planned and prepared in detail prior to disassembly, when the error source is known before, leading to faster regeneration and higher capacity utilization.

Three basic hypotheses for this methodological approach are that, firstly, operation failures inside the combustor or the turbine have an influence on the exhaust jet pattern, secondly, this exhaust jet pattern is so significant that it can be detected by suitable measurement devices, and, thirdly, the connection between the source of a failure inside the engine and the measurable deviation in the exhaust jet can be depicted by numerical simulation. The aspect of measurability (second hypotheses) is currently investigated in detail in (Hartmann and Seume, 2018) and (Hartmann et al., 2016).

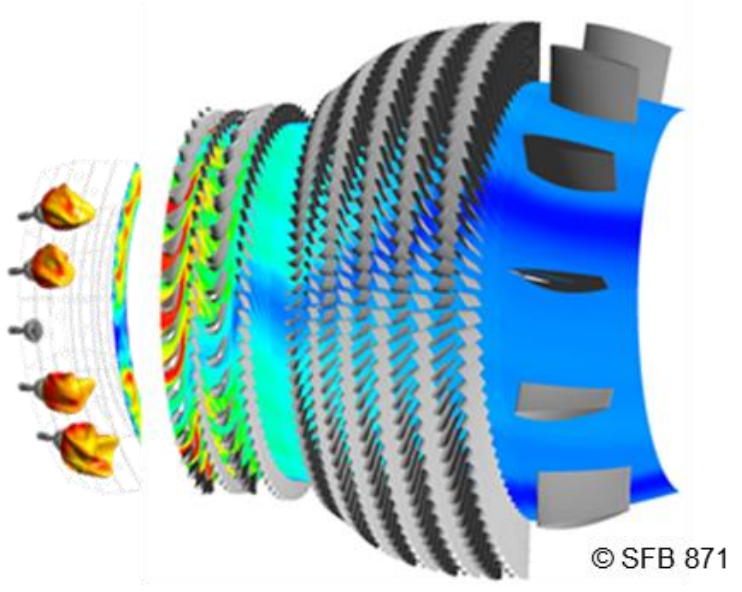

Figure 1: Simulated Propagation of a Streak in the Hot Gas Path of an Aircraft Engine 
In order to investigate the first and the third hypotheses, two steps have been done in recent work. On the one hand, extended studies were made with a pilot scale combustion chamber consisting of eight burners. One of them can be varied in its operation conditions. The results of both experimental and numerical studies were compared, which prove the reliability of this method of failure detection (von der Haar et al., 2016; Hennecke, 2018). On the other hand, the impact of failures on a real engine's combustor is investigated numerically. These results will be described in the current article.

With the mentioned laboratory experiments on the atmospheric eight burner chamber, it is found that defined failures in the combustion chamber lead to detectable temperature and species pattern in the exhaust jet (von der Haar et al., 2016; Hennecke, 2018). Flow and reaction processes were simulated parallelly using three-dimensional numerical simulation methods. The exhaust patterns are in good congruence with those of the experiments and suitable for the prediction of the influence of combustor defects (Hennecke, 2018). These simulations enable to assign a failure inside the combustion chamber to a specific exhaust pattern.

With respect to real engines, also first simulation studies were performed to investigate the path of failure pattern (i.e. a streak with reduced temperature) through the following multistage turbine, showing that inside the turbine the mixing of this failure is rather low, see Figure 1 (Adamczuk and Seume, 2012). Although this fact contradicts the expectation at a first glance, it can be explained by the stream line flow inside a well-designed turbine, where only a small amount of entropy is produced from turbulent mixing. The influence on the processes in the turbine will be investigated in more detail in the ongoing project.

The current work focusses on the processes inside a real size combustion chamber of a turbojet engine. Since an application to real size experiments would be highly expensive as well as difficult to perform, the method of numerical simulation is applied here. It is to mention that further experiments are planned in the ongoing project period within the partner consortium of CRC 871.

\section{NUMERICAL SETUP}

The investigated combustor is part of a commercial twoshaft turbofan aircraft engine with a maximum thrust of about $110 \mathrm{kN}$. The three-dimensional simulations of the reacting flow processes are conducted with the method of computational fluid dynamics (CFD) with additional reaction models within the software ANSYS Fluent 17.2. The current work focuses on the prediction of the influence of combustor defects to determine the turbine inlet conditions for the operation point cruise.

The annular combustion chamber is supplied with fuel and air by 20 spray nozzles, which are shown in Figure 2. The nozzle consists of two different swirl vanes, whereas the fuel will be mixed to the primary air at the end of the inner pipe.

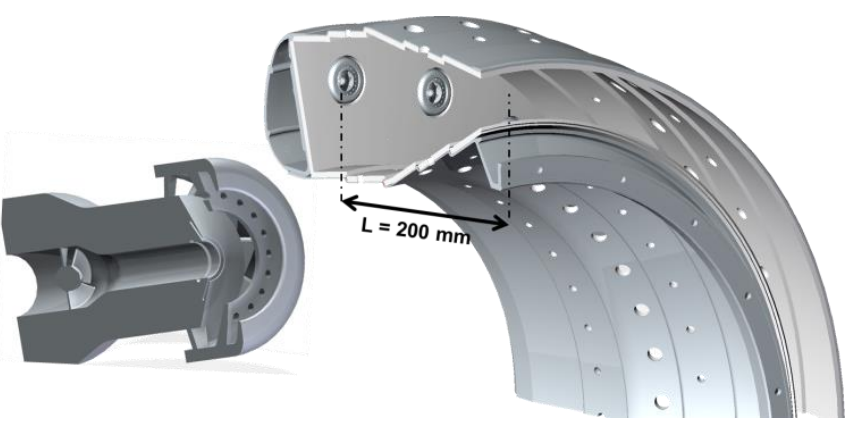

Figure 2: Nozzle and Combustion Chamber

The inner and outer liners of the combustor are protected from the combustion temperatures by injecting cooling air through the film cooling slots on each side. Each liner contains three rows of liner holes for supporting the combustion, the reaction, and cooling processes.

For the numerical simulations a segment of five burners is selected, which represents one quarter segment (Figure 5). Periodic boundary conditions ensure accurate transitions on the cut planes to save computational costs. The diffuser and casing of the combustion chamber are not taking into account. The inlet conditions in the nozzle and the combustion tube are determined based on information about the air distribution from the manufacturer. For the CFD simulation the combustor is resolved with a numerical mesh of approximately 14.5 million tetrahedral cells being locally refined (see Figure 3).
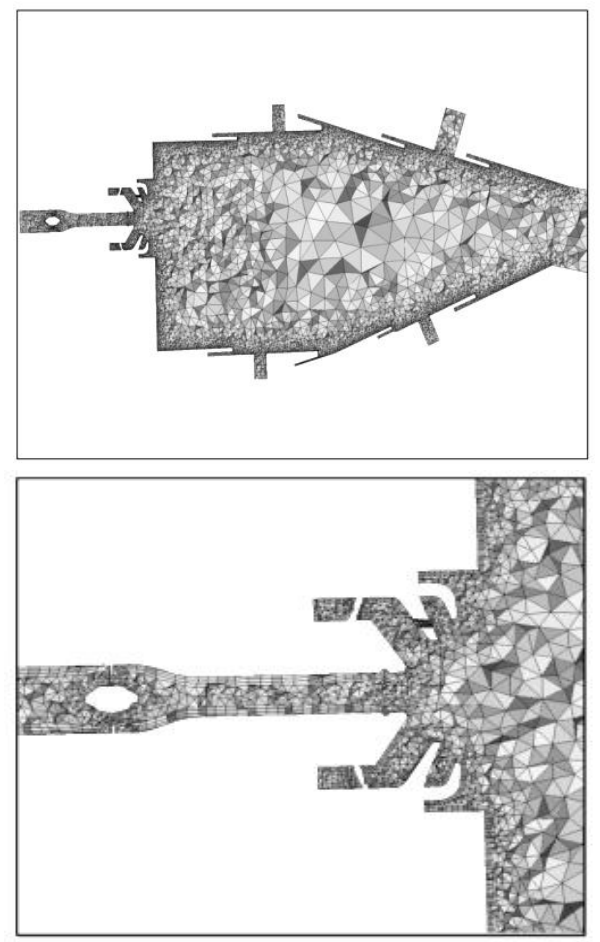

Figure 3: Numerical Grid of the Burning Chamber (top) and a Nozzle (bottom)

Flow and turbulence are determined using the transient SAS model (Menter et al., 2003; Menter and Egorov, 2010). Detailed studies have shown that here a good compromise 
between calculation costs and realistic flow pattern is given also in regions with large scale flow structures (Hennecke, 2018). The reaction process is modelled with a partially premixed combustion model, consisting of the extended coherent flame model for the premixed regime combined with the presumed probability density function (pre-PDF) as a mixture fraction approach for the inhomogeneous fuel-air mixture (Poinsot and Veynante, 2010; Jones and Whitelaw, 1982). Gaseous jet fuel A was chosen as fuel. Within the prePDF approach the fuel influences the thermochemical conditions of the mixture fraction model. It is, however, beyond the possibilities of reaction modelling of such highly turbulent flames, to include all the details of the subreactions (Poinsot and Veynante, 2010).

The processes of spray breakup and evaporation are also neglected because of their additional complexity. This is rationalised due to the idea that this study focusses on the basic possibility to see the influence of burning chamber failures and not on the very details of the burning process. Also the heat transfer of the combustor walls is set to zero. Both are acceptable assumptions for the current study where the focus is laid on the mixing processes inside the burning chamber rather than on the details very near to the burner or the wall.

\section{DEFECT CASES IN THE COMBUSTION CHAMBER}

Possible damages in the combustion chamber are known to occur mainly in three parts of the combustion chamber, the fuel nozzle region, the supply of secondary air through the liner and possibly deformed combustor casings leading to unsymmetrically shifted arrangement between nozzle position and liner. For the fuel nozzle either the fuel supply may be reduced due to clogged fuel lines or the primary air flow can show deviations, for instance from damaged or smutched swirler of the primary air supply. This can lead either to reduced heat release or even to variation in the local air-fuel ratio and strongly modified long and sooting flames, if the swirler fails to hold the turbulent flame in its typical compact form. Possible damage at the inner and outer liner can influence the secondary air distribution, coming, for instance, from changed shape of the bore diameter of the liner holes, e.g. as a result of abrasions or due to high tolerances in the repair processes. Deformation of the liner may be the result of impermissibly high component temperatures and stress. This may cause a faulty positioning of the flame tube and fuel nozzles.

Figure 4 illustrates exemplarily that CFD is an appropriate method to identify causes of damages and to simulate the influences on the flow field further downstream. The picture on the left side shows typical damages (cracks and spalling) on the ceramic tiles caused by temperature stress. On the right side the simulated wall temperatures are shown. It is clearly visible that the highest temperatures occur in the bottom part of the combustion chamber close to the burners. In this area major damages are typically expected. The temperature increases above the liner holes because of the disturbance of the film cooling.

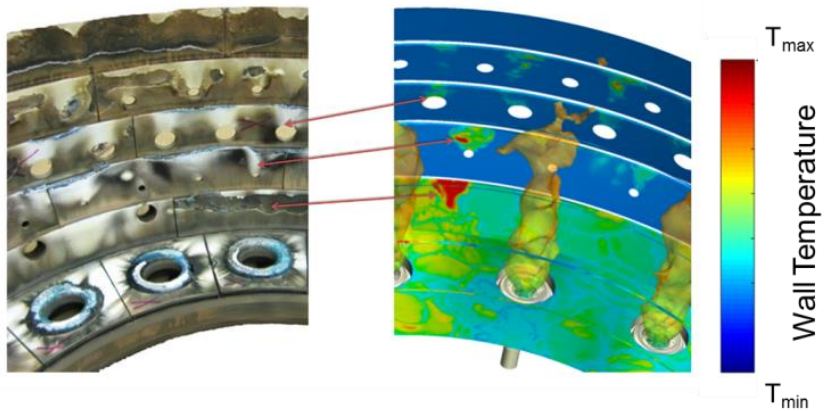

Figure 4: Typical Damages at the Outer Liner (left) and Simulated Wall Temperature

The current work is held generic with respect to the potential failures. It focuses on two types of defects in combustion chambers, each defect varied in two different ways, and their influence on the flow field. Therefore, a total of four cases besides the reference case are examined (see Table 1). In study A, the thermal power of the middle burner is reduced while holding the power of the other burners constant. The power is reduced either to $50 \%$ (DefA-50) or totally shut-down (DefA-0), which represents a blockage of the fuel line. The primary air supply is not changed. The position of the manipulated burner is marked in red (see Figure 5). The following evaluations refer to these basic settings. It may be noted that both cases A are simplified in comparison to stronger variations; for instance a reduced swirl (e.g. broken swirler or closed primary air path) modifies the flame to be much larger than in the reference case with less mixing and increased soot formation. It can be assumed that such a case would be more easily detectable due to the soot emission and possibly significant $\mathrm{CO}$ emission caused by incomplete combustion.

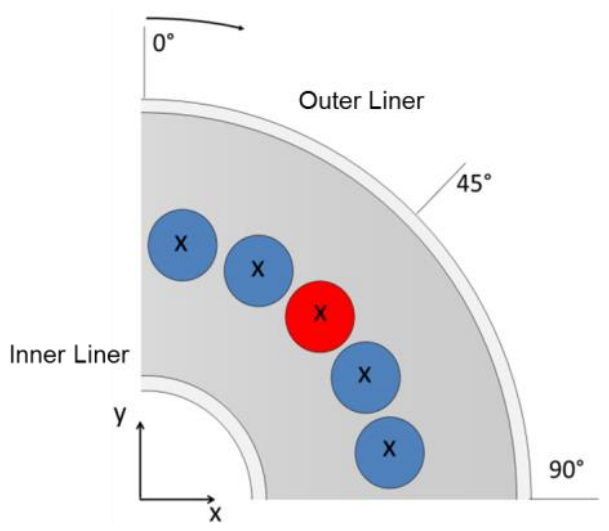

Figure 5: Schematic View of the Burner
Arrangement

Study B represents two simplified assumed cases where the secondary air is provided unsymmetrically to the combustor, possibly being initialized by stress and wear of the liner segments. In the case DefB-IL the air mass flow of the liner holes of the inner liner is assumed to be reduced by $10 \%$ while the missing air mass is added to the liner holes of the outer liner. Also the opposite case is investigated, where 
the air mass flow of the outer liner is reduced by $10 \%$ (DefB-OL) and the flow of the inner liner is increased correspondingly.

\begin{tabular}{l|c|c|c}
\multicolumn{1}{c|}{ Case } & $\dot{\mathbf{m}}_{\text {Jet-A, middle }}$ & $\dot{\mathbf{m}}_{\text {Outer-Liner }}$ & $\dot{\mathbf{m}}_{\text {Inner-Liner }}$ \\
\hline Ref & $100 \%$ & $100 \%$ & $100 \%$ \\
\hline DefA-50 & $50 \%$ & $100 \%$ & $100 \%$ \\
\hline DefA-0 & $0 \%$ & $100 \%$ & $100 \%$ \\
\hline DefB-OL & $100 \%$ & $90 \%$ & $118.1 \%$ \\
\hline DefB-IL & $100 \%$ & $105.5 \%$ & $90 \%$
\end{tabular}

Table 1: Investigated Operation Points

\section{INFLUENCE OF DEFECTS IN A COMBUSTION CHAMBER ON THE COMBUSTOR EXHAUST PLANE}

For the reference case and for the four selected failure cases the three dimensional flow and reaction process is calculated with the mentioned CFD approach with the transient SAS model.

The influence of the reduction of the fuel mass flow (study A) is compared with the reference case in Figure 6. The transient calculations show the 3-dimensional temperature distribution of one time step on the iso-surface of the mixture fraction of $f=0.05$, which can be seen as that area in space where most of the reaction inside these nonpremixed flames takes place. These presentations show the length and position of the main reaction zone inside the flame.

In the reference case the middle burner is operating identically to the other burners. The calculated temperature field shows periodic pattern in the range of 1500 to $2300 \mathrm{~K}$ for the upper region of the combustor. A significant reduction of the flame length and the temperature at the outlet is the result of the calculations of case DefA-50, where the fuel flow rate is reduced to $50 \%$ for the middle burner. Reasons are the decreased impulse of the fuel with the identical mass flow of the secondary air through the liner holes, which limit the combustion zone. In case DefA-0 a total burner failure is shown. Here the influence on the temperature distribution is even stronger visible.

In Figure 7 and Figure 8 the simulated temperature and velocity magnitude distributions are plotted at the outlet plane of the combustion chamber. Even for the reference case the temperature distribution depends on the individual burner, and the temperature pattern is connected to the individual swirl stabilized flames. For the cases with reduced fuel mass flow rate the temperature pattern in the combustor exit plane shows clearly the reduced temperatures in the corresponding exit region of the faulty burner. Also the flow field is affected significantly, showing flow velocities of about 80 to $120 \mathrm{~m} / \mathrm{s}$. For the assumed failures also here the influence is clearly detectable.
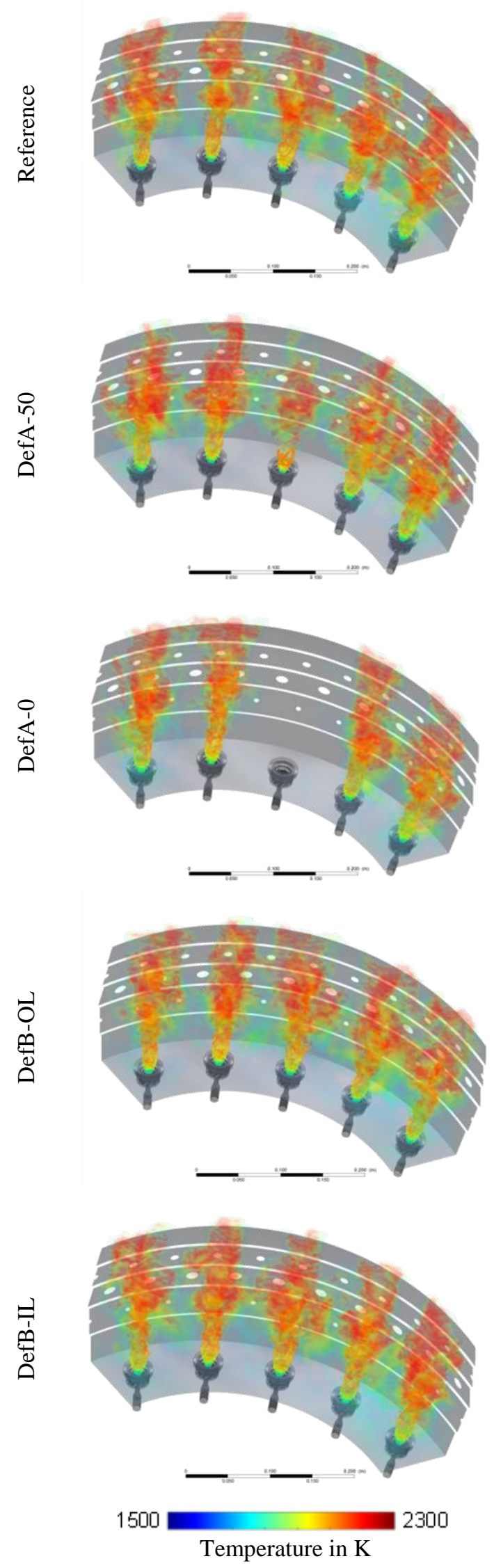

Figure 6: Temperature Distribution for the Reference and the Failure Cases 

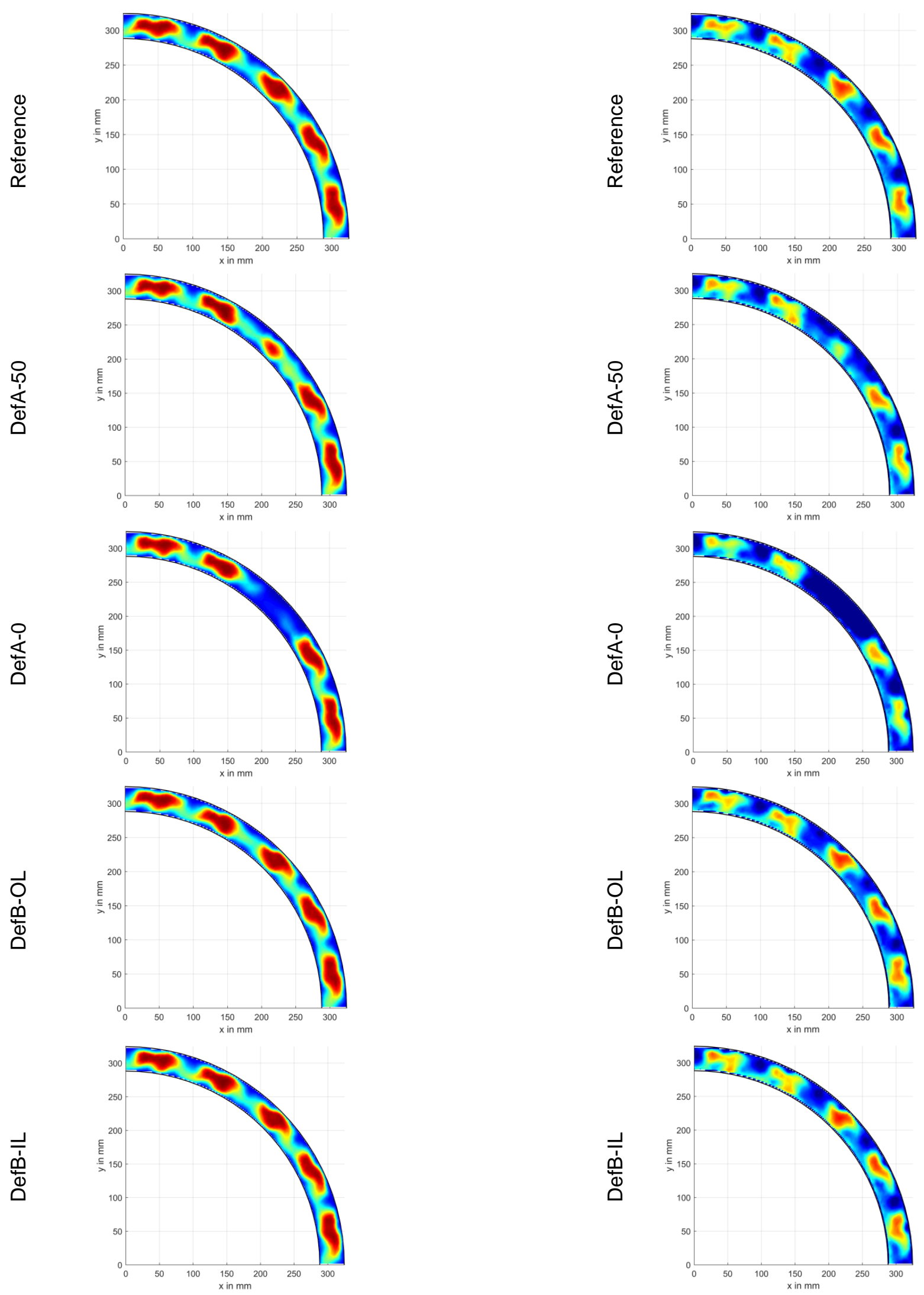

800

Temperature in $\mathrm{K}$

Figure 7: Temperature Profile at the Combustor Outlet Plane

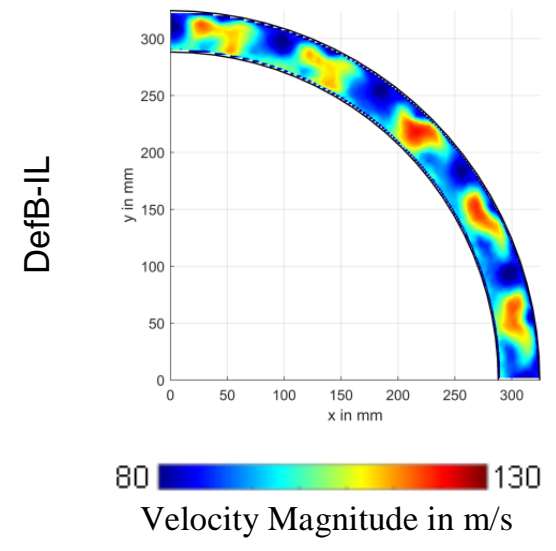

Figure 8: Velocity Magnitude Profile at the Combustor Outlet Plane 
In study B the secondary air in the diffuser casing is manipulated, so that the flow through the liner holes is reduced to $90 \%$ for either all the inner or all the outer liner, while it is increased for the other side. The first expectation is that the flame position will be moved to the inner or outer side of the burner. The simulations do not show this expected effect. No significant deviations from the reference case can be seen concerning temperature and velocity distribution for the investigated operation points at the exit plane. Obviously, the modified secondary air flow has rather low influence on the flame position. This may be explained by the strongly swirling flow around the rather localized flames in such way, that the secondary air is entrained into the swirl but is not moving the flames. As can be seen from Figure 7 and Figure 8 neither the exit plane distribution of temperature nor of velocity are varied so significantly that an observation of this failure type would be detectable.

Figure 9 shows the radially averaged temperature distribution at the outlet plane as a function of the angle (see also Figure 5). The temperature pattern of the outer four burners is not significantly affected by the failures. The temperature profiles are similar for all cases with maximum temperatures between $1800 \mathrm{~K}$ and $1900 \mathrm{~K}$, showing the rather homogenous combustion process and no interactions with the exhaust gases of the manipulated burner in the middle. The reduction of the power (study A) leads to a significant reduction of the temperature of about $850 \mathrm{~K}$ (DefA-0) or $350 \mathrm{~K}$ (DefA-50) at the combustor exit plane.

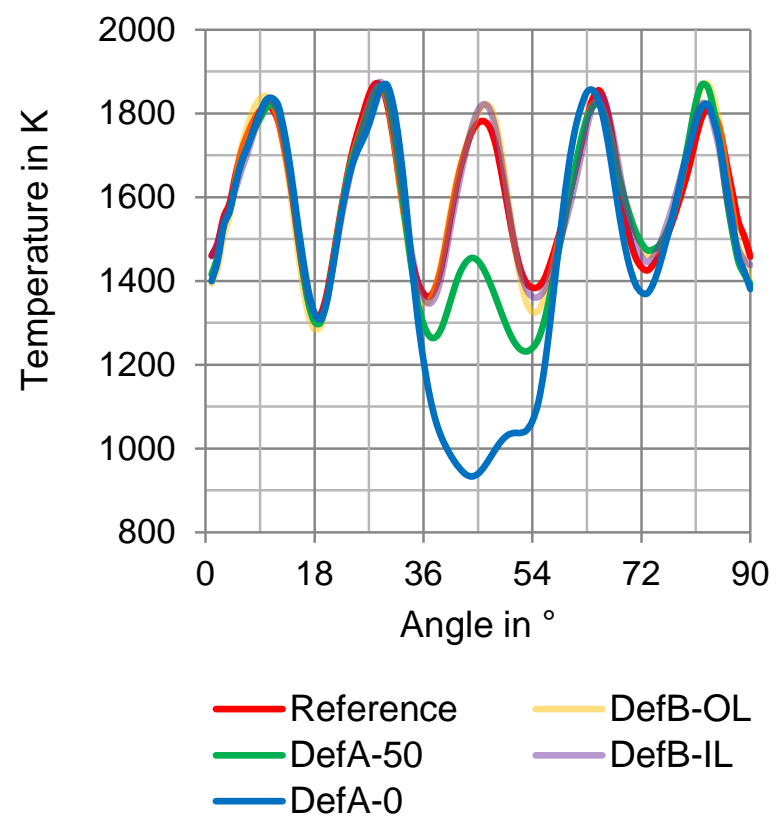

\section{Figure 9: Radially Averaged Temperature Distribution at the Combustor Outlet}

The influences of the considered defects in study B are very small compared to those in study A. The temperature profiles are slightly equalized by up to $50 \mathrm{~K}$ in the cases $\mathrm{B}$ OL and B-IL, possibly being a result of more entrained air in the flame region due to the more unsymmetrical secondary air supply. However, it is quite obvious that a measurement of the exhaust temperature pattern will not be so sensitive to detect these pattern cases.

In Figure 10 the radially averaged velocity magnitude distributions are plotted for the reference and the investigated defect cases. The velocities above the non-manipulated burners illustrate larger interactions between the burners concerning the velocity distribution. The defects in study A lowers the maximum velocity magnitude at $45^{\circ}$ from $105 \mathrm{~m} / \mathrm{s}$ to $70 \mathrm{~m} / \mathrm{s}$ (DefA-0) or $93 \mathrm{~m} / \mathrm{s}$ (DefA-50). The lower exhaust flow velocity is due to the reduced mass flow and gas expansion of this burner.

The defects of study B have much smaller influence on the original velocity distribution. For the defect case DefBOL the minimum velocity at $36^{\circ}$ and $54^{\circ}$ decreases by $3 \mathrm{~m} / \mathrm{s}$.

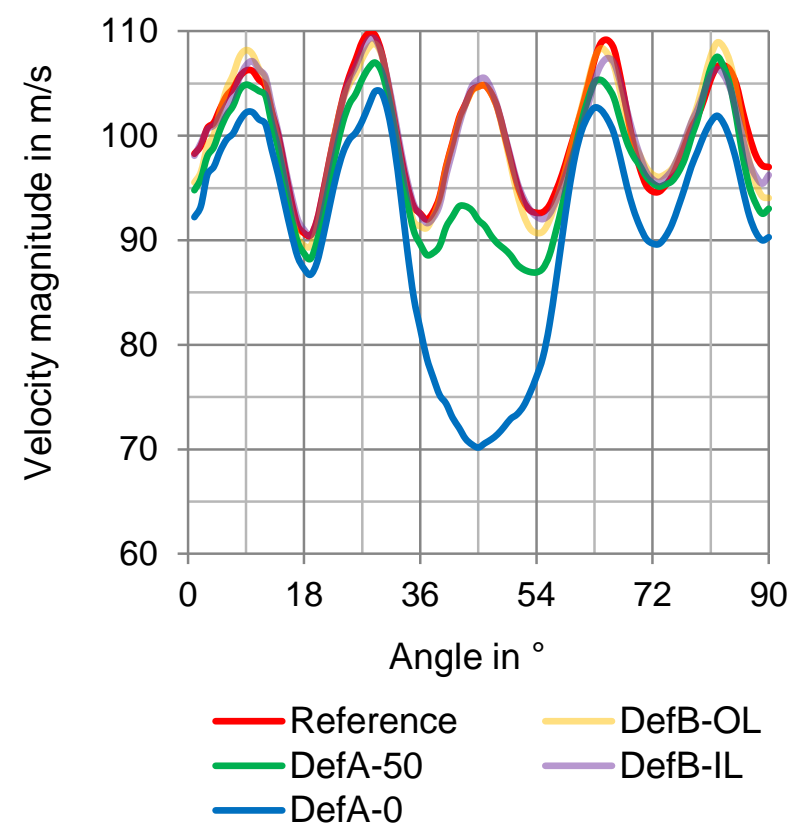

\section{Figure 10: Radially Averaged Velocity Magnitude Distribution at the Combustor Outlet}

Figure 11 shows the relative temperature deviation of the investigated defect cases from the reference case at the combustor outlet. The relative deviation is defined in Equation 1:

$$
\delta=\frac{x_{r e f}-x_{d e f}}{x_{r e f}} \cdot 100 \%
$$

whereas $x_{\text {ref }}$ and $x_{\text {ref }}$ are quantities of the reference and of the failure case.

The influence of the defect cases is limited to the area above the manipulated burner, as seen before. The maximum relative deviation of the radially averaged temperature distribution is $47 \%$ (DefA-0) or $21 \%$ (DefA-50). The relative deviation of the non-manipulated burners is about 6 $\%$. The influences of the defects of study B are lower than 6 $\%$ for the whole plot, wherefore these cases are not distinguishable from the reference case. 


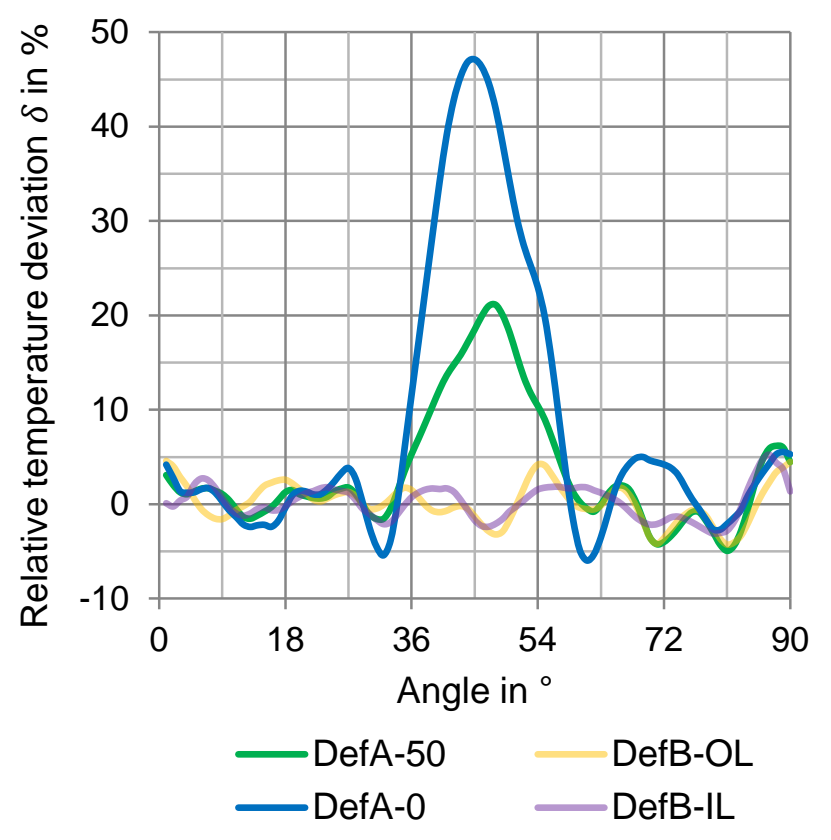

\section{Figure 11: Relative Temperature Deviation from the Reference Case at the Combustor Outlet}

In Figure 12 the relative velocity magnitude deviations are plotted. The maximum relative deviation is $33 \%$ (DefA$0)$ or $13 \%$ (DefA-50). In the edge region the velocity deviation is about $6 \%$, as well. In this diagram the influences of the defects of study B are again not clearly distinguishable from the reference case.

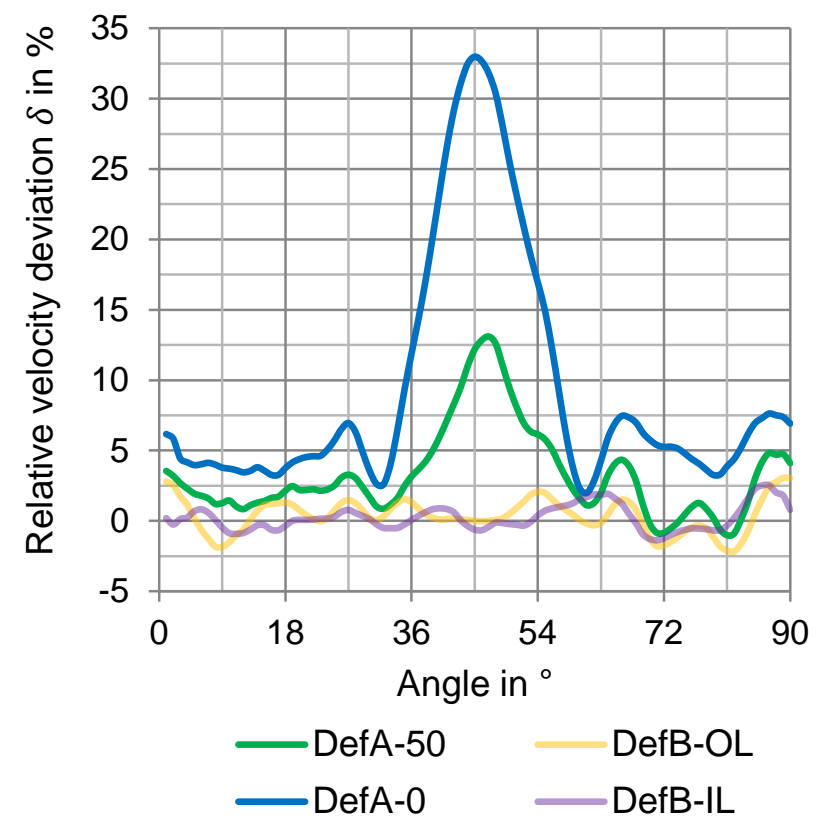

\section{Figure 12: Relative Velocity Deviation from the Reference Case at the Combustor Outlet}

The shown study is focussed on the combustion chamber and the resulting pattern on its exit. It is most likely that for an application of the overall failure detecting method the subsequent turbine has an additional influence on the mixing process of potentially inhomogeneous temperature, species or velocity pattern. In ongoing work these effects will be investigated in more detail with an approach to analyse the turbulent diffusive mixing process in such situations. The flow channels inside the turbine may either enhance or may reduce the mixing process. It seems likely that the latter is more dominant as the passage through the enclosed flow channels in the turbine elements suppress large scale turbulent mixing (Figure 1). When the mixing of inhomogeneous temperature or species or velocity pattern in a turbine section can be quantified, a judgement is expected about the possible minimal size of deviations from the turbine exit plane which could be detectable.

Another future aim is the subsequent implementation of an automatic defect classification system based on the exhaust profile measurement. For the classification of the corresponding defect, a low impact of mixing and a unique pattern is necessary, as otherwise a faulty defect assignment could occur.

\section{CONCLUSIONS}

Numerical studies were conducted on a segment of an annular combustion chamber of an aircraft engine, where the influences of defined failures on the temperature and velocity pattern at the combustor exit plane are investigated. Besides the reference case the operation condition of one of the swirl burners has been varied in two steps (Study A) or the secondary air flow distribution has been shifted to the inner or outer liner side by about $10 \%$ (Study B).

The investigations discussed in this work show that the lateral turbulent mixing between the jet engine flames is low and the calculated temperature and velocity profiles at the outlet show compact structures of each burner.

In study A the fuel mass flow of one burner was reduced from the references state to $50 \%$ and to $0 \%$, representing a defective fuel supply. The variations are leading to a distinguishable modified pattern at the exhaust plane of the combustion chamber concerning the temperature and velocity distribution. This confirms the formulated first and third hypotheses for the investigated situation. In study B the distribution of the secondary air flow was changed. The influences are nearly invisible at the exit plane of the combustor and, therefore, will most likely not be detectable behind the exit of the turbine.

Although the current work on the failure detection of real size engine combustors is done only numerically, as experiments are very expensive and difficult, they are based on earlier work on a model ring burning chamber with eight burners, where one could be operated in defined "faulty" mode. Here, both simulation and experiments were conducted and led to rather congruent results. This gives an indication that also for the more complex geometry in real jet engines the simulation results will give at least a qualitative indication of reality. It is planned within the collaborative research work CRC 871 that real size tests will be performed with failures in the next research period.

In future work also the turbulent dispersion process of failure structures in the exhaust gas path will be investigated 
in more detail. This is another essential step to determine the limit of detectable failure structures behind the aircraft engine. As the flow is straightened between the stator and rotor blade passage with an expected suppression of the large scale turbulence, it can be possible that some class of failure pattern inside the aircraft engine might be measurable in the exhaust gas pattern without the need to disassemble the jet engine. If this method was successful, it would allow the development of on-condition-based maintenance concepts in aviation.

\section{NOMENCLATURE}

\section{Abbreviations}

$\begin{array}{ll}\text { CFD } & \text { Computational Fluid Dynamics } \\ \text { CRC } & \text { Collaborative Research Center } \\ \text { RANS } & \text { Reynolds-Averaged Navier-Stokes } \\ \text { SAS } & \text { Scale-Adaptive Simulation }\end{array}$

\section{Symbols}

$\begin{array}{ll}\delta & \text { relative deviation } \\ f & \text { mixture fraction } \\ x_{\text {ref }} & \text { physical quantity (reference case) } \\ x_{\text {def }} & \text { physical quantity (defect case) }\end{array}$

\section{ACKNOWLEDGMENTS}

This project is funded by the German Research Foundation (DFG - Deutsche Forschungsgemeinschaft) within the framework of the Collaborative Research Centre CRC 871, subproject A4 "Influence of Combustion Chamber Defects on the Exhaust Jet" and A6 "Impact of Mixing on the Signature of Combustor Defects".

\section{REFERENCES}

[1] Adamcuk R., and Seume J. R. (2012). Time Resolved Full-Annulus Computations of a Turbine with Inhomogeneous Inlet Conditions. International Journal of Gas Turbine, Propulsion and Power Systems, 4, No 2, pp 1-7 [2] CRC871 (2018). Collaborative Research Center: "Regeneration of Complex Capital Goods". Leibniz University Hannover, www.sfb871.de

[3] Hartmann U. and Seume J.R. (2018). Automated Condition Evaluation of Hot-Gas Path Components of Jet Engines through Exhaust Jet Analysis. Proceedings of the ASME Turbo Expo 2018, Oslo, Norway. GT2018-75384

[4] Hartmann U., Hennecke C., Dinkelacker F. and Seume J. R. (2016). Automatic Detection of Defects in a Swirl Burner Array Through an Exhaust Jet Pattern Analysis, J. Eng. Gas Turbines Power 139 (3).

[5] Hennecke C. (2018). Methodik einer Zustandsbeurteilung von Triebwerksbrennkammern, Dissertation, Leibniz Universität Hannover, TEWISS-Verlag: Berichte aus dem ITV, Band 1/2018.

[6] Jones W. P. and Whitelaw J. H. (1982). Calculation Methods for Reacting Turbulent Flows: A Review. Combustion and Flame, Vol. 48, pp. 1-26
[7] Menter F. R., Kuntz M. and Bender, R. (2003). A Scale-Adaptive Simulation Model for Turbulent Flow Predictions., 41st Aerospace Sciences Meeting and Exhibit, Reno, Nevada, USA

[8] Menter F. R. and Egorov Y. (2010). The ScaleAdaptive Simulation Method for Unsteady Turbulent Flow Predictions. Part 1: Theory and Model Description, Flow, Turbulence and Combustion, Vol. 85, No. 1, pp. 113-138

[9] Poinsot T. and Veynante D. (2012). Theoretical and Numerical Combustion, Third Edition. Cerfacs, Toulouse.

[10] von der Haar H., Hartmann U., Hennecke C., Dinkelacker F. and Seume J. R. (2016). Defect Detection in an Annular Swirl-Burner-Array by Optical Measuring Exhaust Gases. Proceedings of ASME Turbo Expo 2016, Seoul, South Korea. GT2016-57847 